\title{
Osteopathic Approach to Treatment of Carpal Dysfunction: The Carpal Mobilization Technique
}

Thomas R. Mehner, OMS IV; Drew D. Lewis, DO

From the Des Moines

University College of Osteopathic Medicine in lowa.

Financial Disclosures: None reported.

Support: This video was produced by Des Moines University.

Address correspondence to Drew D. Lewis, DO Des Moines University College of Osteopathic Medicine, 3200 Grand Avenue, Des Moines, IA 50312-4104.

Email: drew.d.lewis@dmu.edu

Video link: https://youtu.be/ op3sWk-OuMU

Submitted May 4, 2020; revision received July 17, 2020;

accepted August 7, 2020.

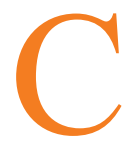
arpal bone motion dysfunction, or pain upon motion or palpation, is a common finding in patients with carpal tunnel syndrome (CTS). Multiple diagnostic and therapeutic techniques can be used to address somatic dysfunction related to CTS. Here, we provide an example of a simple and efficient diagnostic evaluation followed by osteopathic manipulative treatment (OMT) for carpal bone somatic dysfunction (video).

The classic symptoms of carpal tunnel syndrome are paresthesia, numbness, or burning in the thumb and the lateral 2.5 digits. ${ }^{1}$ If compression of the median nerve in the carpal tunnel is not alleviated, wasting of the thenar eminence can occur, along with weakness of the thumb.

The carpal tunnel comprises the transverse carpal ligament on the anterior surface and the carpal bones on the posterior surface. The transverse carpal ligament runs from the hamate and pisiform bones medially to the scaphoid and trapezium laterally. ${ }^{2}$ The carpal tunnel contains 10 structures: 4 tendons of the flexor digitorum profundus, 4 tendons of the flexor digitorum superficialis, the flexor policis longus tendon, and the median nerve, the latter of which is the most sensitive structure. ${ }^{2}$

The causes of CTS are multifactorial, but the condition is generally, as mentioned earlier, a compression of the median nerve. Changes in the anatomy of the carpal tunnel, such as a lunate dislocation, can lead to increased pressure within the tunnel and compression of the median nerve. ${ }^{1}$ The "gold standard" for confirming CTS is electrodiagnostic testing, which can determine the presence and severity of nerve damage, measure the effects of treatment, and help rule out other conditions. ${ }^{3}$ Ultrasonography may be used to provide complementary results to electrodiagnostic testing. ${ }^{3}$
Many studies ${ }^{4-8}$ have been performed to evaluate the clinical efficacy of OMT on patients with CTS. Research by Sucher ${ }^{4-7}$ and Sucher et $\mathrm{al}^{8}$ showed OMT to be effective at reducing CTS symptoms, improving median nerve function as measured with nerve conduction studies, and increasing carpal tunnel dimensions as measured with ultrasound and magnetic resonance imaging. A pilot study by Burnham et $\mathrm{al}^{9}$ found that OMT significantly improved CTS symptoms and function after a 6-week treatment course, but the authors did not find significant changes in median nerve function on electrodiagnostic testing or ultrasonography.

Addressing somatic dysfunction of the carpal bones is important regardless of the underlying cause of CTS. Somatic dysfunctions of the wrist are related to a restriction of the gliding motions of the individual carpal bones as well as the carpal bones on the radius. ${ }^{10}$ Carpal bones can be displaced toward the palm, therefore narrowing the carpal tunnel and contributing to congestion or compression on the median nerve. ${ }^{10}$ Improving the motion of the carpal bones and encouraging the proper alignment increases space in the carpal tunnel, decreases the pressure on the median nerve, and increases axoplasmic, blood, and lymphatic flow to the area. ${ }^{10}$

There are multiple ways to diagnose somatic dysfunction of the carpal bones. Assessing for elements of tissue texture change, asymmetry, restricted range of motion, or tenderness (TART) can guide the physician to a diagnosis. One diagnostic approach is to perform a general assessment, evaluating for restriction of motion between the carpal bones by taking the wrist into flexion, extension, abduction, and adduction. ${ }^{10}$ Performing minor motion glides by moving individual carpal bones in the anterior and posterior directions can be useful for identification of 
carpal restriction. As the motion can be quite subtle, comparison with the unaffected limb can also be helpful to appreciate any asymmetry. Throughout the assessment of the carpal bone restriction, tissue texture changes may be noted in addition to any tenderness of the wrist.

The carpal mobilization technique addresses carpal bone restriction of motion in any direction. With the patient in the supine position, with a pronated forearm (with the patient's palm facing the ground), the physician places their thumb pads on the proximal carpal row while their fingertips are in contact with the thenar and hypothenar eminences of the affected hand (video). The physician then brings the wrist through full flexion and extension while adding radial and ulnar gliding. An important component of the treatment is when the patient's wrist is brought into full extension the physician's fingers, which are applying lateral traction to the thenar and hypothenar eminences and simultaneously applying a distraction pressure to gently separate the carpal bones. Gentle articulation is often appreciated by the patient and physician. The physician reassesses by examining the carpal bone motion after treatment, looking for increased range of motion and resolution of tissue texture changes, asymmetry, or tenderness. Contraindications to this technique include distal radioulnar joint instability (positive piano key sign) and acute fracture of any carpal bones, radius, or ulna.

While the treatment in this video focuses on somatic dysfunction of the wrist region, the osteopathic approach to treating a patient with CTS is typically much broader. Diagnosis and treatment should address other areas of potential somatic dysfunction, especially along the course of the nerve from spinal root levels through the distal nerve. This should be particularly focused on areas of potential median nerve entrapment, such as the pronator teres or the flexor digitorum-sublimis bridge, and other key areas including: the cervical and thoracic spine; thoracic inlet region (first ribs, scalene muscles, and clavicle); pectoralis minor and upper rib cage; shoulder; elbow; and forearm. Specific key somatic dysfunctions to evaluate and address include inhalation dysfunction of ribs, extended clavicle dysfunction, posterior radial head, or radial head tenderpoint.

There are multiple diagnostic and treatment techniques to address carpal bone somatic dysfunction as it relatese to CTS. The technique presented in the video is directed at management of restricted motion between the carpal bones as part of a complete treatment for CTS (doi: 10.7556/jaoa.2020.120).

\section{Acknowledgments}

We thank Ryan McMunn, OMS IV, for being the patient-model in the video accompanying this article.

\section{References}

1. Siu G, Jaffe JD, Rafique M, Weinik MM. Osteopathic manipulative medicine for carpal tunnel syndrome. J Am Osteopath Assoc. 2012;112(3):127-139.

2. Moore KL, Agur AMR, Dalley AF. Upper Limb. In: Essential Clinical Anatomy. 5th ed. Philadelphia, PA: Lippincott Williams \& Wilkins; 2015:458-460.

3. Zhao M, Burke DT. Median neuropathy (carpal tunnel syndrome). In: Frontera WR, Silver JK and Rizzo TD. Essentials of physical medicine and rehabilitation. 4th ed. Elsevier; 2019:191-196.

4. Sucher BM. Myofascial release of carpal tunnel syndrome. J Am Osteopath Assoc. 1993;93(1):92-94, 100-101.

5. Sucher BM. Myofascial manipulative release of carpal tunnel syndrome: documentation with magnetic resonance imaging. J Am Osteopath Assoc. 1993;93(12):1273-1278

6. Sucher BM. Palpatory diagnosis and manipulative management of carpal tunnel syndrome. J Am Osteopath Assoc. 1994;94(8):647-663.

7. Sucher BM. Palpatory diagnosis and manipulative treatment of carpal tunnel syndrome, part 2: "double crush" and thoracic outlet syndrome. J Am Osteopath Assoc. 1995;95(8):471-479.

8. Sucher BM, Hinrichs RN, Welcher RL, Quiroz LD, St Laurent BF, Morrison BJ. Manipulative treatment of carpal tunnel syndrome: biomechanical and osteopathic intervention to increase the length of the transverse carpal ligament, part 2: effect of sex differences and manipulative "priming". J Am Osteopath Assoc. 2005;105(3):135-143.

9. Burnham T, Higgins DC, Burnham RS, Heath DM. Effectiveness of osteopathic manipulative treatment for carpal tunnel syndrome: a pilot project. J Am Osteopath Assoc. 2015;115(3):138-148. https://doi.org/ 10.7556/jaoa.2015.073

10. Heinking KP. Osteopathic Considerations in Sports Medicine: Uppe Extremities. In: Seffinger MA, executive ed. Foundations of Osteopathic Medicine. 4th ed. Wolters Kluwer; 2018:1488-1496.

(c) 2020 American Osteopathic Association 